\title{
CARACTERÍSTICAS VOCAIS DE GÊMEOS MONOZIGÓTICOS
}

\author{
Vocal features of monozygotic twins
}

\author{
Carla Aparecida Cielo (1), Rosane Agustini (2), Leila Susana Finger ${ }^{(3)}$
}

\section{RESUMO}

Tema: características vocais de gêmeos monozigóticos e sua relação com a normalidade. Procedimentos: participaram da pesquisa dois pares de gêmeos adultos, um par de cada sexo, que realizaram avaliação otorrinolaringológica, auditiva, vocal perceptivo-auditiva e acústica da fonte glótica. Os dados foram apresentados de forma descritiva e discutidos comparativamente com os dados de normalidade da literatura e do programa utilizado. Para testar a diferença entre as médias e a normalidade, utilizou-se o teste t de Student (significância 5\%). Resultados: não houve diferenças quanto ao ataque vocal, articulação, loudness, ressonância, modo e tipo respiratórios em ambos os pares de gêmeos monozigóticos avaliados, estando os resultados dentro dos padrões de normalidade. Houve sinais de hipertensão muscular relacionada à produção vocal em ambos os pares, o tipo de voz foi considerado discretamente alterado em ambos os pares, e houve diferença quanto ao tempo máximo de fonação dos gêmeos. Todas as medidas acústicas relacionadas à frequência fundamental estiveram dentro dos padrões de normalidade do Multi Dimensional Vocal Program. Conclusão: os resultados encontrados no presente estudo se apresentaram dentro da normalidade e parecem seguir a tendência daquelas descritas em estudos que avaliaram gêmeos monozigóticos.

DESCRITORES: Fonação; Voz; Qualidade da Voz; Gêmeos Monozigóticos

\section{INTRODUÇÃO}

A voz humana é uma das formas exclusivas de identidade pessoal. Muitas vezes, a voz é comparada a uma impressão digital, sugerindo que não existem duas vozes exatamente iguais ${ }^{1}$. Pode-se inferir que a voz de um indivíduo é única, partindose do pressuposto de que as medidas vocais de um indivíduo se devem a aspectos anatômicos, fisiológicos e psicológicos, não podendo existir dois seres humanos que possuam exatamente as mesmas características vocais ${ }^{2-4}$.

Há diversos estudos relatando a grande similaridade de voz e de fala em gêmeos monozigóticos,

(1) Fonoaudióloga; Professora do Curso de Fonoaudiologia e do Programa de Pós-Graduação em Distúrbios da Comunicação Humana da Universidade Federal de Santa Maria, UFSM, Santa Maria, RS; Doutora em Linguística Aplicada pela Pontifícia Universidade Católica do Rio Grande do Sul.

(2) Fonoaudióloga; Clínica Rosane Agustini, RS; Especialista em Reabilitação em Fonoaudiologia - Ênfase em Voz pelo Instituto Metodista de Educação e Cultura do Rio Grande do Sul.

(3) Fonoaudióloga; Prefeitura Municipal de Portão, RS; Mestre em Distúrbios da Comunicação Humana pela Universidade Federal de Santa Maria.

Conflito de interesses: inexistente devido ao seu desenvolvimento psicológico e à suposição de que estiveram expostos aos mesmos fatores ambientais ${ }^{2,5,6}$. O estudo de gêmeos idênticos é do interesse de diversas áreas do conhecimento, uma vez que permite a exploração do papel que os fatores genéticos e ambientais exercem sobre o desenvolvimento ${ }^{2,5-13}$.

A semelhança entre a voz de gêmeos pode ser explicada por fatores geneticamente determinados, como a similaridade da anatomia da laringe, assim como elementos psicológicos igualmente herdados que ajudam a determinar o comportamento vocal ${ }^{4,5}$. É importante destacar também a influência dos fatores ambientais no desenvolvimento da voz desde o nascimento ${ }^{5}$.

Entretanto, quando os gêmeos envelhecem (ou são separados) e experimentam maior independência, suas vozes estão sujeitas a diferentes influências. Isto conduz às diferenças nas características não genéticas de suas vozes, como o hábito de tabagismo, bem como a separação geográfica que pode conduzir à variação do dialeto. Tais diferenças já têm sido investigadas e relatadas em diversos estudos fonéticos ${ }^{2}$.

Tendo em vista que há poucos estudos publicados relatando as características vocais de gêmeos 
monozigóticos 4-6,12-14, em especial na literatura nacional, este trabalho teve como objetivo descrever as características vocais de um par de gêmeos adultos monozigóticos de cada sexo e discutir comparativamente com os dados de normalidade da literatura e do programa utilizado.

\section{APRESENTAÇÃO DOS CASOS}

A presente pesquisa, descritiva e apresentada sob a forma de Estudo de Caso tem o intuito de descrever e analisar as características vocais de gêmeos monozigóticos.

Os critérios de inclusão do estudo foram: gêmeos monozigóticos, com base em critérios subjetivos em relação ao aspecto da zigosidade ${ }^{15}$ foram observados quanto ao aspecto físico, levando-se em consideração a cor dos olhos e cabelos, tipo de cabelo, forma do rosto, formato do nariz, dentes, estatura, e outras características que mostrassem a igualdade física intrapar. A opinião dos irmãos gêmeos quanto à sua zigosidade foi considerada, bem como a opinião de familiares, em especial da mãe; adesão ao termo de consentimento livre e esclarecido (TCLE).

Os critérios de exclusão para este estudo foram: queixas vocais, como rouquidão, fadiga vocal, falhas na voz ou ardência na garganta, visto que estes são sintomas sugestivos de algum tipo de alteração vocal orgânica ou comportamental, podendo interferir nos resultados da pesquisa; patologias laríngeas, pois distúrbios no nível laríngeo poderiam comprometer os resultados da avaliação; apresentar história pregressa de doenças neurológicas, endocrinológicas, psiquiátricas ou gástricas ${ }^{16}$, que poderiam influenciar a performance vocal ou o entendimento das ordens durante as avaliações; alterações hormonais decorrentes de gravidez ou período menstrual e pré-menstrual, coletadas por meio de anamnese aberta ${ }^{17}$; estar com gripe e/ou alergias respiratórias ${ }^{18}$, porque ambos podem causar edema nas pregas vocais; hábitos de etilismo e tabagismo, já que esses agentes são agressivos à laringe e podem originar problemas vocais orgânicos; alterações auditivas, pois elas podem modificar o auto-monitoramento da voz, comprometendo a qualidade vocal; ter realizado tratamento fonoaudiológico e/ou otorrinolaringológico prévios, para descartar a possibilidade de que o sujeito tivesse qualquer patologia vocal (mesmo já tratada).

Após a aplicação do TCLE, foram realizadas algumas avaliações com o objetivo de descartar possíveis alterações que pudessem interferir nos resultados da pesquisa.

Os sujeitos foram submetidos à avaliação otorrinolaringológica para verificação de possíveis alte- rações ou patologias laríngeas. Essa avaliação foi realizada em clínica particular, com uso de laringoscopia com fibra ótica, sendo o laringoscópio rígido introduzido por meio da boca do paciente. As avaliações foram gravadas em vídeo e em CD. Os gêmeos foram submetidos, também, à triagem auditiva para detectar possíveis alterações. Essa triagem foi realizada por meio de uma varredura de tons puros nas frequências de 500, 1000, 2000 e $4000 \mathrm{~Hz}$ a $25 \mathrm{~dB}$, somente pela via aérea, com audiômetro VSC da Auditec, em cabine acusticamente tratada.

Os participantes foram entrevistados individualmente, sendo solicitadas algumas informações e um depoimento sobre a própria voz: como o próprio entrevistado percebia sua voz, comentários de outras pessoas sobre a sua voz, e uma comparação com a voz do seu irmão. Essa entrevista foi gravada e utilizada na avaliação como amostra de fala espontânea e para o levantamento de dados pessoais, investigação de possíveis alterações que pudessem impedir a participação dos sujeitos na pesquisa, bem como para avaliação de questões ligadas a habilidades gerais de comunicação e psicodinâmica vocal para posterior comparação entre os pares de gêmeos.

Após o processo de seleção, com base nos critérios de exclusão, os voluntários que apresentaram alteração em alguma das avaliações foram descartados da pesquisa e encaminhados para avaliações mais completas. Durante este processo, houve a exclusão de um par de gêmeos monozigóticos do sexo feminino devido a alterações laríngeas; de um par de irmãs gêmeas, em que uma das irmãs realizava fonoterapia vocal; e de dois pares do sexo masculino que não conseguiram dispor de tempo para realizar todas as avaliações.

Atenderam aos critérios de inclusão e de exclusão e realizaram a coleta de dados, quatro irmãos gêmeos, sendo um par do sexo feminino com idade de 28 anos, e um par do sexo masculino com idade de 20 anos.

Em sala silenciosa ${ }^{3}$, a anamnese e a coleta de todas as amostras de voz dos participantes foram gravadas em minidisc Walkman Sony modelo MZ-R700, por meio de microfone Shure, modelo Dynamic Cardioid Microphone 8800. O microfone foi posicionado a uma distância de $15 \mathrm{~cm}^{3,19-21}$ em frente à boca do entrevistado, que estava em pé com os braços estendidos ao longo do corpo. Para a realização das análises perceptivo-auditiva e acústica, as amostras de voz, armazenadas em mini-disc, foram digitalizadas num computador Pentium III e gravadas no formato wave em CD, o que permitiu a análise das medidas relacionadas à frequência fundamental por meio do programa 
Multi Dimensional Vocal Program da Kay PENTAX (MDVP): frequência fundamental - fO $(\mathrm{Hz})$; desviopadrão da frequência fundamental - STD $(\mathrm{Hz})$; média da frequência fundamental - MfO $(\mathrm{Hz})$; frequência fundamental mais alta - fhi $(\mathrm{Hz})$; frequência fundamental mais baixa $-\mathrm{flo}(\mathrm{Hz})^{3,21,22}$.

Para a análise vocal acústica dessas medidas, utilizou-se a amostra da vogal /a/ sustentada em altura e intensidade habituais, utilizando-se um tempo mínimo de três segundos, sendo excluído o início e o término da emissão para que o ataque vocal ou possível decréscimo final da intensidade não interferissem na análise ${ }^{3,21,22}$. As amostras da vogal /a/ dos sujeitos foram quantizadas em $25 \mathrm{kHz}$ e 16bits para esta análise.

Para a análise vocal perceptivo-auditiva e das medidas de tempo máximo de fonação (TMF), foram utilizadas as gravações em CD da amostra de fala espontânea da anamnese, as medidas de (TMF), que incluem os TMF de vogais (/a/, /i/, /u/), relação s/z e TMF da contagem de números ${ }^{3}$, e a gravação da emissão dos dias da semana, da contagem numérica de 100 a um, e dos meses do ano. Os TMF foram cronometrados e os sujeitos eram orientados a, em pé, inspirar pelo nariz e emitir vogais (/a/, /i/, /u/) e os fonemas /s/ e /z/ em tom, intensidade e velocidade habituais, sustentando-os durante o tempo máximo possível durante a mesma expiração ${ }^{3,21,22}$, por três vezes, sendo escolhida a maior sustentação ${ }^{21,22}$. Na avaliação do TMF da contagem numérica, o procedimento foi o mesmo da avaliação dos TMF de vogais e fricativas, solicitando-se que o sujeito realizasse a contagem a partir do numeral um até o final de uma expiração sem inspirar nesse tempo ${ }^{3}$. Ainda, para a avaliação perceptivo-auditiva, foi solicitado aos sujeitos que emitissem os dias da semana, os meses do ano e que contassem de 100 até um.

Por meio da avaliação perceptivo-auditiva, realizada por duas juízas, fonoaudiólogas especialistas em voz, foi traçado o perfil vocal de cada participante, identificando-se: tipo de voz; tipo de ataque vocal; tipo de articulação; pitch; foco vertical de ressonância; e loudness. Os julgamentos foram realizados individualmente, de forma independente, sendo considerada a concordância entre as juizas. No caso de divergências, uma terceira juíza avaliava as vozes da mesma forma, sendo, então, considerada a predominância entre as avaliadoras. Também foram avaliados o modo e o tipo respiratórios durante a fala; a média dos TMF das vogais /a/, /i/, /u/; a relação entre os TMF de /s/ e /z/; e o resultado da contagem de números.

O presente estudo foi previamente aprovado pelo Comitê de Ética em Pesquisa da instituição de origem (23081.008439/2007-16/0087.0.243.000-07) e teve início após a assinatura do TCLE pelos participantes, conforme a norma 196/96 da Comissão Nacional de Ética em Pesquisa.

Para testar a existência de diferença significativa entre as médias e a normalidade, utilizou-se o teste t de Student, adotando-se um nível de significância igual a $5 \%$.

\section{RESULTADOS}

\section{Gêmeos do sexo feminino}

Os resultados das avaliações otorrinolaringológicas das duas irmãs foram considerados normais, não sendo observada qualquer alteração laríngea. $O$ resultado da triagem auditiva indicou limiares auditivos normais para as duas irmãs. Com relação às informações relevantes da anamnese, as irmãs referiram que frequentemente eram confundidas ao telefone.

Os resultados da análise vocal perceptivoauditiva e acústica das medidas relacionadas à frequência fundamental das gêmeas se encontram nas Tabelas 1 e 2.

\section{Gêmeos do sexo masculino}

Os resultados das avaliações otorrinolaringológicas dos dois irmãos foram considerados normais, não sendo observada qualquer alteração laríngea. $O$ resultado da triagem auditiva indicou limiares auditivos normais para ambos. Com relação aos dados relevantes da anamnese, observou-se o fato de o gêmeo 2 praticar esportes regularmente e referir utilizar mais a voz no curso de Jornalismo.

Os resultados da análise vocal perceptivo-auditiva e acústica das medidas relacionadas à frequência fundamental dos gêmeos se encontram nas Tabelas 3 e 4.

\section{DISCUSSÃO}

As medidas de $\mathrm{fo}$ fornecem dados relacionados ao comprimento, à tensão, à rigidez e à massa das pregas vocais, e sua relação com a pressão aérea subglótica ${ }^{3}$. Neste estudo, a diferença da f0 das irmãs foi de 16,824Hz, não apresentando diferença significativa (Tabela 2 ) em relação à normalidade proposta pelo MDVP, além de estar dentro da faixa tomada como padrão no Brasil ${ }^{2,3,4,6,14,22}$. O mesmo ocorreu em relação aos gêmeos do sexo masculino, cujos valores de fo tiveram uma variação de $5 \mathrm{~Hz}$ entre o par (Tabelas 2 e 4) ${ }^{21}$. Nas demais medidas relacionadas à f0 (STD; Mf0; fhi; flo) também não foi observada diferença estatisticamente significante das medidas em relação à normalidade (Tabelas 2 e 4) ${ }^{21,22}$. 
Tabela 1 - Resultados da análise vocal perceptivo-auditiva e das medidas de tempo máximo de fonação das gêmeas

\begin{tabular}{|c|c|c|c|c|c|}
\hline & Gêmea 1 & Gêmea 2 & $\begin{array}{l}\text { Variação das } \\
\text { medidas entre } \\
\text { as gêmeas }\end{array}$ & $\begin{array}{c}\text { Valores de } \\
\text { normalidade }\end{array}$ & p \\
\hline Tipo de voz & $\begin{array}{l}\text { discretamente } \\
\text { soprosa }\end{array}$ & $\begin{array}{l}\text { discretamente } \\
\text { soprosa }\end{array}$ & - & - & - \\
\hline Ataque vocal & isocrônico & isocrônico & - & - & - \\
\hline Articulação & precisa & precisa & - & - & - \\
\hline $\begin{array}{l}\text { Modo } \\
\text { respiratório }\end{array}$ & misto & misto & - & - & - \\
\hline $\begin{array}{l}\text { Tipo } \\
\text { respiratório }\end{array}$ & $\begin{array}{c}\text { costodiafragmá } \\
\text { tico }\end{array}$ & $\begin{array}{c}\text { costodiafragmá } \\
\text { tico }\end{array}$ & - & - & - \\
\hline Loudness & adequado & adequado & - & - & - \\
\hline $\begin{array}{l}\text { Foco de } \\
\text { ressonância }\end{array}$ & equilibrado & equilibrado & - & - & - \\
\hline $\begin{array}{l}\text { Média de } \\
\text { emissão de } \\
\text { vogais /a, i, u/ } \\
\text { (segundos) }\end{array}$ & 13,86 & 12,85 & 1,01 & $14,00^{3}$ & 0,4229 \\
\hline Relação s/z & $\begin{array}{c}0,74 \\
\text { hipertensão } \\
\text { laríngea }\end{array}$ & 0,99 & 0,25 & 0,80 a $1,20^{3}$ & 0,4755 \\
\hline $\begin{array}{l}\text { Contagem de } \\
\text { números } \\
\text { (segundos) }\end{array}$ & 15,75 & 17,32 & 1,57 & - & - \\
\hline $\begin{array}{l}\text { Comparação } \\
\text { entre a média } \\
\text { das vogais e a } \\
\text { contagem } \\
\text { numérica } \\
\text { (segundos) }\end{array}$ & 1,89 & $\begin{array}{c}5,47 \\
\text { hipertensão do } \\
\text { mecanismo de } \\
\text { fala }\end{array}$ & 3,58 & $\begin{array}{l}1 \text { a } 3 \text { segundos maior } \\
\text { do que a média das } \\
\text { vogais testadas } \\
\text { valor }>4 \text { segundos } \\
\text { sugere hipertensão do } \\
\text { mecanismo de fala }{ }^{3}\end{array}$ & - \\
\hline
\end{tabular}

LEGENDA: Teste t-student; * significância estatística em relação à normalidade

Tabela 2 - Medidas vocais acústicas relacionadas à frequência fundamental das gêmeas

\begin{tabular}{|c|c|c|c|c|c|}
\hline $\begin{array}{l}\text { Medidas } \\
\text { acústicas }\end{array}$ & Gêmea 1 & Gêmea 2 & $\begin{array}{l}\text { Variação das } \\
\text { medidas entre } \\
\text { as gêmeas }\end{array}$ & $\begin{array}{c}\text { Valores de } \\
\text { normalidade do } \\
\text { Multi Dimensional } \\
\text { Vocal Program }\end{array}$ & $p$ \\
\hline fo $(\mathrm{Hz})$ & 216,587 & 199,763 & 16,824 & 145,22 a 243,27 & 0,3458 \\
\hline STD $(\mathrm{Hz})$ & 2,265 & 2,039 & 0,226 & 2,722 & 0,1209 \\
\hline MfO (Hz) & 216,564 & 199,742 & 16,822 & 241,080 & 0,1592 \\
\hline fhi $(\mathrm{Hz})$ & 222,732 & 208,202 & 14,530 & 252,724 & 0,1226 \\
\hline flo $(\mathrm{Hz})$ & 210,818 & 191,477 & 19,341 & 234,861 & 0,1778 \\
\hline
\end{tabular}

LEGENDA: Teste t student; * significância estatística em relação à normalidade; frequência fundamental - f0 (Hz); desvio-padrão da frequência fundamental - STD $(\mathrm{Hz})$; média da frequência fundamental - $\mathrm{MfO}(\mathrm{Hz})$; frequência fundamental mais alta - fhi $(\mathrm{Hz})$; frequência fundamental mais baixa - flo $(\mathrm{Hz})$. 
Tabela 3 - Resultados da análise vocal perceptivo-auditiva e das medidas de tempo máximo de fonação dos gêmeos

\begin{tabular}{|c|c|c|c|c|c|}
\hline & Gêmeo 1 & Gêmeo 2 & $\begin{array}{l}\text { Variação das } \\
\text { medidas entre } \\
\text { os gêmeos }\end{array}$ & $\begin{array}{c}\text { Valores de } \\
\text { normalidade }\end{array}$ & $\mathbf{p}$ \\
\hline Tipo de voz & $\begin{array}{l}\text { discretamente } \\
\text { rouca }\end{array}$ & $\begin{array}{l}\text { discretamente } \\
\text { rouca }\end{array}$ & - & - & - \\
\hline Ataque vocal & alternado & alternado & - & - & - \\
\hline Articulação & precisa & precisa & - & - & - \\
\hline $\begin{array}{l}\text { Modo } \\
\text { respiratório }\end{array}$ & misto & misto & - & - & - \\
\hline Tipo respiratório & misto & misto & - & - & - \\
\hline Loudness & adequado & adequado & - & - & - \\
\hline $\begin{array}{l}\text { Foco de } \\
\text { ressonância }\end{array}$ & equilibrado & equilibrado & - & - & - \\
\hline $\begin{array}{l}\text { Média de } \\
\text { emissão de } \\
\text { vogais /a, i, u/ } \\
\text { (segundos) }\end{array}$ & 8,9 & 35,6 & 26,7 & $20,00^{3}$ & - \\
\hline Relação s/z & $\begin{array}{c}0,77 \\
\text { hipertensão } \\
\text { laríngea }\end{array}$ & 0,93 & 0,16 & 0,80 a $1,20^{3}$ & 0,8937 \\
\hline $\begin{array}{l}\text { Contagem de } \\
\text { números } \\
\text { (segundos) }\end{array}$ & 28,9 & 44 & 15,1 & - & 0,3119 \\
\hline $\begin{array}{l}\text { Comparação } \\
\text { entre a média } \\
\text { das vogais e a } \\
\text { contagem } \\
\text { numérica } \\
\text { (segundos) }\end{array}$ & $\begin{array}{c}20 \\
\text { hipertensão no } \\
\text { mecanismo de } \\
\text { fala }\end{array}$ & $\begin{array}{l}8,4 \\
\text { hipertensão no } \\
\text { mecanismo de } \\
\text { fala }\end{array}$ & 11,6 & $\begin{array}{l}1 \text { a } 3 \text { segundos maior } \\
\text { do que a média das } \\
\text { vogais testadas } \\
\text { valor }>4 \text { segundos } \\
\text { sugere hipertensão do } \\
\text { mecanismo de fala }{ }^{3}\end{array}$ & - \\
\hline
\end{tabular}

LEGENDA: Teste t-student; * significância estatística em relação à normalidade

Tabela 4 - Medidas vocais acústicas relacionadas à frequencia fundamental dos gêmeos

\begin{tabular}{lccccc}
\hline $\begin{array}{l}\text { Medidas } \\
\text { acústicas }\end{array}$ & Gêmeo 1 & Gêmeo 2 & $\begin{array}{c}\text { Variação das } \\
\text { medidas entre } \\
\text { os gêmeos }\end{array}$ & $\begin{array}{c}\text { Valores de } \\
\text { normalidade do Multi } \\
\text { Dimensional Vocal } \\
\text { Program }\end{array}$ & $\mathbf{p}$ \\
\hline fO $(\mathrm{Hz})$ & 125,183 & 130,390 & 5,207 & $<145,22$ & 0,0944 \\
STD $(\mathrm{Hz})$ & 0,830 & 1,239 & 0,409 & 1,349 & 0,3682 \\
MfO $(\mathrm{Hz})$ & 125,177 & 130,378 & 5,201 & 141,743 & 0,1172 \\
fhi $(\mathrm{Hz})$ & 127,582 & 134,405 & 6,823 & 150,080 & 0,1127 \\
flo $(\mathrm{Hz})$ & 123,338 & 127,118 & 3,780 & 140,418 & 0,0788 \\
\hline
\end{tabular}

LEGENDA: Teste t student; * significância estatística em relação à normalidade; frequência fundamental - f0 (Hz); desvio-padrão da frequência fundamental - STD $(\mathrm{Hz})$; média da frequência fundamental - MfO $(\mathrm{Hz})$; frequência fundamental mais alta - fhi $(\mathrm{Hz})$; frequência fundamental mais baixa - flo $(\mathrm{Hz})$. 
Os resultados apresentados vão ao encontro daqueles descritos em um estudo ${ }^{13}$ que avaliou a f0 durante a leitura de um texto padronizado em língua alemã de 30 pares de gêmeas monozigóticas, 30 pares de dizigóticas, e de um grupo de controle de pares de não irmãs, entre 15 e 29 anos de idade. Observou-se alta correlação entre a f0 das gêmeas monozigóticas, menor correlação entre as dizigóticas, e nenhuma correlação entre a f0 dos pares do grupo controle, evidenciando a base genética da f0, uma vez que a literatura aponta que ela está relacionada ao comprimento, rigidez e tensão das pregas vocais, geneticamente determinados. Por outro lado, a variação intra-individual da fo da fala no referido estudo foi muito similar entre as gêmeas monozigóticas e dizigóticas, o que pode indicar que nesse aspecto não é possível discernir a influência da disposição genética e a participação do ambiente $2,8,13$.

Reforçando essas afirmações, um estudo mostrou que a média da f0 da fala, entre gêmeos, apresenta maior semelhança do que entre indivíduos não relacionados, pareados ao acaso na população em geral, o que poderia indicar a presença de componente genético na variação dessa medida ${ }^{14}$.

De modo geral, o que se pôde verificar na avaliação vocal perceptivo-auditiva das gêmeas vai ao encontro das semelhanças descritas na literatura ${ }^{4-6}$. Os resultados das avaliações sugerem que as gêmeas apresentaram hipertensão muscular durante a fonação, evidenciada por duas medidas diferentes: a relação s/z para a gêmea 1 , relacionada ao excesso de tensão em nível glótico e a comparação entre a média das vogais e a contagem numérica para a gêmea 2 , relacionada à excessiva tensão no mecanismo de fala (Tabela 1), apesar do tipo de voz ter sido julgado como discretamente soproso para ambas (Tabela 1). Os resultados da média dos TMF das vogais e da relação $s / z$ foram estatisticamente semelhantes aos valores propostos como normalidade para a população brasileira (Tabela 1) ${ }^{3,22}$.

Os dados da avaliação perceptivo-auditiva dos gêmeos (Tabela 3) da presente pesquisa seguem a tendência observada na avaliação das gêmeas, inclusive no que diz respeito à hipertensão muscular durante a fonação, evidenciada pela relação s/z para gêmeo 1 e contagem numérica para ambos (Tabela 3). Pôde-se observar diferença dos tempos máximos de fonação (TMF) entre os gêmeos, discrepância que pode ser explicada por fatores ambientais, uma vez que gêmeo 2 referiu praticar esportes regularmente, além de utilizar mais a voz no curso de Jornalismo, o que poderia justificar seus elevados tempos máximos de fonação, o que vai ao encontro da literatura ${ }^{4}$ uma vez que fatores ambientais influenciam qualidade vocal, performance vocal, usos incorretos, expressões vocais, canto e habilidade musical desde o nascimento.

Quanto às características ressonantais, a literatura aponta que o número de harmônicos e o espectro de frequência também refletem maiores semelhanças em gêmeos monozigóticos quando comparados a gêmeos dizigóticos ou a pares de mesma idade e sexo ${ }^{5,13}$. Neste estudo, a ressonância vocal foi analisada perceptivo-auditivamente e considerada equilibrada, dentro da normalidade, nos quatro sujeitos, mostrando semelhança entre os dois pares de gêmeos monozigóticos avaliados. Resultado que converge com a avaliação perceptivo-auditiva do ataque vocal, da articulação, do modo e do tipo respiratórios durante a fala, bem como do pitch e loudness que também se mostraram adequados e semelhantes entre os pares (Tabelas 1 e 3).

Na literatura $2,5,6,13$, também se afirma a estreita similaridade entre as vozes de gêmeos monozigóticos e se reforça a necessidade de que mais estudos precisam ser desenvolvidos no que tange à identificação vocal de gêmeos idênticos, uma vez que ocorre um desenvolvimento psicológico muito semelhante nestes casos. No entanto, quando gêmeos monozigóticos têm experiências independentes, suas vozes estão sujeitas a diferentes influências externas e consequentemente, podem se tornar menos semelhantes, o que pode ter ocorrido no caso do gêmeo 2 em relação ao gêmeo 1 quanto aos TMF.

Quanto ao tipo de voz, o julgamento perceptivoauditivo evidenciou igualdade entre cada par de gêmeos, mas discreta alteração em relação à normalidade, uma vez que as gêmeas apresentaram voz discretamente soprosa e os gêmeos apresentaram voz discretamente rouca ${ }^{3}$.

De forma geral, verificou-se que as características vocais perceptivo-auditivas e relacionadas à f0 dos pares de gêmeos monozigóticos avaliados no presente estudo se apresentaram dentro da normalidade proposta pela literatura e pelo programa utilizado, com exceção da característica de hipertensão muscular do par de gêmeas e da diferença entre os TMF dos gêmeos, da hipertensão muscular durante a fonação, evidenciada pela relação s/z para o gêmeo 1 e pela contagem numérica para ambos.

Em pesquisa ${ }^{6} \mathrm{com}$ o objetivo de verificar as similaridades vocais em gêmeos idênticos, dez pares do sexo feminino e cinco pares do sexo masculino, todos monozigóticos, de origem belga, a proporção correta de identificação foi de $82 \%$ para as vozes femininas e de $63 \%$ para as vozes masculinas. A avaliação foi realizada por um grupo 
de dez ouvintes (estudantes de fonoaudiologia), sendo solicitado que relacionassem a voz dos pares de gêmeos. As vozes gravadas de cada par foram reunidas a uma terceira voz, formando um trio de vozes. Concluiu-se que havia maior semeIhança entre as vozes de gêmeos idênticos do que com as vozes sem relação genética e que o pitch vocal possivelmente contribuiu para a correta identificação dos gêmeos.

\section{CONCLUSÃO}

Este estudo permitiu concluir que não houve diferenças quanto ao ataque vocal, articulação, loudness, ressonância, modo e tipo respiratórios em ambos os pares de gêmeos monozigóticos avaliados, apresentando-se dentro dos padrões de normalidade. Houve sinais de hipertensão muscular relacionada à produção vocal em ambos os pares, o tipo de voz foi considerado discretamente alterado em ambos os pares, e houve diferença quanto ao TMF dos gêmeos.

Todas as medidas acústicas relacionadas à f0 utilizadas nesta pesquisa estiveram dentro dos padrões de normalidade do programa e da literatura.

Os resultados encontrados no presente estudo parecem seguir a tendência daquelas descritas em estudos que avaliaram gêmeos monozigóticos.

\begin{abstract}
Background: vocal features of monozygotic twins and their relation with the normative data for unrelated peers. Procedures: the sample was composed of two pairs of adult twins, one pair of each gender had undergone otorhinolaryngologic, auditive, vocal auditory perceptual and acoustic evaluations. Data was showed in a descriptive way and discussed comparatively with the normative data for unrelated peers on literature and on the applied program. The test $t$ of Student (significance $5 \%$ ) was used to test the differences between the average and the normative values. Results: there were no differences related to voice onset, articulation, loudness, resonance, breathing type and mode in both evaluated monozygotic twin pairs, and they were adequate. There were signs of muscular hypertension related to vocal production in both pairs, the type of voice was considered slightly altered in both pairs and there was a difference regarding the twin's Maximum Phonation Time. All the acoustic measurements were in accordance to the normative patterns of Multi Dimensional Vocal Program. Conclusion: the measurements found in this study seem to follow the trend of those described in studies that evaluated monozygotic twins.
\end{abstract}

KEYWORDS: Phonation; Voice; Voice Quality; Twins, Monozygotic

\section{REFERÊNCIAS}

1. Machado MAMP, Aldrighi JM, Ferreira LP. Os sentidos atribuídos à voz por mulheres após a menopausa. Saúde Pública. 2005; 39(2):261-9.

2. Ariyaeeinia A, Morrison C, Malegaonkar A, Black $S$. A test of the effectiveness of speaker verification for differentiating between identical twins. Sci Justice. 2008; 48(4):182-6.

3. Behlau M, Madazio G, Feijó D, Pontes P. Avaliação de voz. In: Behlau M. Voz: o livro do especialista. Vol 1. Rio de Janeiro: Revinter; 2001. p. $85-245$.

4. Van Lierde KM, Vinck B, De Ley S, Clement G, Van Cauwenberge P. Genetics of vocal quality characteristics in monozygotic twins: a multiparameter approach. J Voice. 2005; 19(4):511-8.

5. Debruyne F, Decoster W, van Gijsel A,
Vercammen J. Speaking fundamental frequency in monozygotic and dizygotic twins. J Voice. 2002; 16(4):466-71.

6. Decoster W, van Gijsel A, Vercammen J, Debruyne F. Voice similarity in identical twins. Acta Otorhinolaryngol Belg. 2001; 55(1):49-55.

7. Barbetta NL, Panhoca I, Zanolli ML. Aspectos fonoaudiológicos e pediátricos na linguagem de gêmeos monozigóticos. Rev Paul Pediatr. 2008; 26(3):265-70.

8. Barbetta NL, Panhoca I, Zanolli ML. Gêmeos monozigóticos: revelações do discurso familiar. Rev Soc Bras Fonoaudiol. 2008; 13(3):267-71.

9. Weber DE, Vares MA, Mota HB, Keske-Soares M. Desenvolvimento do sistema fonológico de gêmeos monozigóticos com desvio fonológico: correlação a fatores genéticos e ambientais. Rev. CEFAC. 2007; 9(1):32-9. dx.doi.org/10.1590/ S1516-18462007000100005 
10. Leite RA, Stivanin L, Oliveira CCC, Rondina C, Matas CG, Scheuer Cl. Funcionamento da via auditiva e da linguagem em gêmeas monozigóticas com características de mutismo seletivo. Rev Neurociências. 2005; 13(2):74-9

11. Ferreira AT, Silva MMA, Silva L, Merighi LBM, Miranda AM, De-Vitto LPM, et al. Desempenho comunicativo em trigêmeos prematuros. Rev. CEFAC. 2008; 10(1):15-21. dx.doi.org/10.1590/ S1516-18462008000100003

12. Ryalls J, Shaw H, Simon M. Voice onset time production in older and younger female monozygotic twins. Folia Phoniat Logopaed. 2004; 56(3):165-9.

13. Flach $M$, Schwickardt $H$, Steinert $R$. Zur Frage des Einflusses erblicher Faktoren auf den Stimmklang (Zwillingsuntersuchungen). Folia Phoniatr. 1968; 20:369-78.

14. Przybyla BD, Horii Y, Crawford M. Vocal fundamental frequency in a twin sample: looking for a genetic effect. J Voice. 1995; 6(3):261-6.

15. Beiguelman B. O estudo de gêmeos [livro on line] [acesso em 28 set 2007]. Disponível em: http:// www.desvirtual.com/bbeiguel/GEMEOS.PRN.pdf 16. Kelchner LN, Horne J, Lee L, Klaben B, Stemple JC, Adam S, et al. Reliability of speech-language pathologist and otolaryngologist ratings of laryngeal signs of reflux in an asymptomatic population using the reflux finding score. J Voice. 2007; 21(1):92-100.
17. Figueiredo LC, Gonçalves MIR, Pontes A, Pontes P. Estudo do comportamento vocal no ciclo menstrual: avaliação perceptivo-auditiva, acústica e auto-perceptiva. Rev Bras Otorinolaringol. 2004; 70(3):331-9.

18. Paes C, Vieira J, Leonel T, Cunha DA. O impacto da respiração oral no comportamento vocal. J Bras Fonoaudiol. 2005; 5(23):417-23.

19. Lima MFB, Camargo ZA, Ferreira LP. Qualidade vocal e formantes das vogais de falantes adultos da cidade de João Pessoa. Rev. CEFAC. 2007; 9(1):99-109. dx.doi.org/10.1590/ S1516-18462007000100013

20. Godino-Llorente Jl, Osma-Ruiz V, Sáenz-Lechón N, Cobeta-Marco I, González-Herranz R, RamírezCalvo C. Acoustic analysis of voice using WPCVox: a comparative study with Multi Dimensional Voice Program. Eur Arch Otorhinolaryngol. 2008; 265(4): 465-76.

21. Beber BC. Características vocais acústicas de homens com voz e laringes normais. [dissertação]. Santa Maria (RS): Universidade Federal de Santa Maria; 2009.

22. Schwarz K. Modificações laríngeas e vocais produzidas pelo som vibrante lingual [dissertação]. Santa Maria (RS): Universidade Federal de Santa Maria; 2006. http://dx.doi.org/10.1590/s1516-18462010005000003

RECEBIDO EM: 03/02/2009

ACEITO EM: 15/10/2009

Endereço para correspondência:

Leila Susana Finger

Rua João Neves da Fontoura, 716/404

São Leopoldo - RS

CEP: 93010-050

E-mail: leilafiner@yahoo.com.br 